\title{
Paisajes costeros: la construcción del patrimonio balneario en Río de Janeiro y Viña del Mar*
}

\section{Coastal landscapes: the construction of seaside heritage in Rio de Janeiro and Viña del Mar}

DOI: $10.46814 / \operatorname{lajdv3n3-050}$

Recebimento dos originais: 01/05/2021

Aceitação para publicação: 31/06/2021

\section{Macarena Cortés Darrigrande}

Doutora em Arquitetura e Estudos Urbanos, Pontificia Universidade Católica do Chile Facultad de Arquitectura, Diseño y Estudios Urbanos. Pontificia Universidad Católica de Chile El Comendador 1916 - Of. 3, Providencia, Santiago de Chile.

E-mail: mmcortes@uc.cl

\section{Thaise Gambarra Soares}

Candidata a Doutora em Arquitetura e Estudos Urbanos, Pontifícia Universidad Católica de Chile. Mestre em Arquitetura e Urbanismo, Universidade Federal da Bahia.

Facultad de Arquitectura, Diseño y Estudios Urbanos. Pontificia Universidad Católica de Chile

El Comendador 1916, Providencia, Santiago de Chile.

E-mail: tgambarra@uc.cl

\section{RESUMEN}

Los bordes costeros generan un paisaje compuesto por un patrimonio construido y natural que determinan condiciones interesantes en la reflexión actual del Patrimonio. Este trabajo propone un acercamiento comparativo entre Viña del Mar y Rio de Janeiro, que permita entender sus diferencias y las consecuencias actuales de los proyectos desarrollados en la conformación patrimonial del borde costero. Las iniciativas desarrolladas apuntan a propuestas de índole y escalas diversas, pero en ambas, las condiciones legislativas se cruzan con los intereses públicos y privados, incidiendo en la toma de decisiones sobre el área más apreciada de la ciudad y en su configuración actual.

Palabras clave: Balneario, Patrimonio, Costanera, Fachada Marítima, Rio de Janeiro, Viña del Mar.

\begin{abstract}
The coastal edges generate a landscape composed of a built and natural heritage that determine interesting conditions in the current reflection on heritage. This paper proposes a comparative approach between Viña del Mar and Rio de Janeiro, in order to understand their differences and the current consequences of the projects developed in the heritage conformation of the coastal edge. The developed initiatives point to proposals of diverse nature and scales, but in both, the legislative conditions intersect with public and private interests, influencing the decision making on the most appreciated area of the city and its current configuration.
\end{abstract}

Key Words: Seaside; Heritage; Waterfront; Waterfront; Rio de Janeiro; Viña del Mar.

\footnotetext{
* Trabajo desarrollado en el marco del proyecto Redes 2017 "Discusión teórica y construcción histórica. El patrimonio arquitectónico de la modernidad en Chile y Brasil" financiado por CONICYT como reflexión de la investigación en desarrollo en el Programa de Doctorado en Arquitectura y Estudios Urbanos de la Pontificia Universidad Católica de Chile y financiado por CONICYT - PCHA/Doctorado Nacional/ 2015 Folio N21151456.
} 


\section{INTRODUCCIÓN}

El trabajo propone reflexionar sobre las diversas condiciones que componen los encuentros de los bordes costeros con centros urbanos litorales. Dichos encuentros, generan un paisaje compuesto por un patrimonio edilicio construido y otro natural, que determinan condiciones particularmente interesantes en la reflexión amplia de lo que hoy se entiende por patrimonio cultural ${ }^{1}$.

El objetivo es poner en relación dos ejemplos emblemáticos en la conformación urbana del litoral, cómo son las conurbaciones de Valparaíso - Viña del Mar y Río de Janeiro - Copacabana. Ambas fueron desarrollándose a lo largo del borde costero, como límite natural y como espacio cultural significativo en su definición arquitectónica. Ello provocó una constante superposición de: planes, proyectos, legislaciones y condiciones de protección, que ayudaron a construir las condiciones urbanas específicas de dichos balnearios. Estas acciones se centraron preferentemente en la accesibilidad pública a la playa, la legitimación del espacio público de calidad a lo largo de la costanera y la fachada marítima, y la protección del entorno natural y sus condiciones ecológicas.

Las diversas acciones emprendidas desde principios del siglo XX en ambos países y hasta la actualidad, muestran la creciente valorización del borde costero como espacio de ocio, veraneo, y turismo, que reforzaron la importancia pública de la playa.

El trabajo propone un acercamiento comparativo, que permite entender las diferencias entre ambos ejemplos y sus consecuencias actuales en la conformación patrimonial del borde costero. Las iniciativas llevadas a cabo en ambos casos apuntan a propuestas de índole y escalas diversas, pero en ambos, las condiciones legislativas, se cruzan con los intereses tanto públicos como privados, que inciden en la toma de decisiones sobre el área más apreciada de la ciudad y en su configuración actual (Figura 1 y 2).

\section{CONDICIONES FUNDACIONALES DE LAS CIUDADES DE RÍO DE JANEIRO Y VIÑA} DEL MAR

Río de Janeiro es un caso muy particular, que pasó desde una ciudad predominantemente portuaria hasta el siglo XIX, enclavada en el interior de la bahía de Guanabara, hacia una ciudad atlántica y reconocidamente balnearia en la actualidad. Este cambio estuvo inserto en un contexto de intensas transformaciones sociales, políticas y urbanas $^{2}$, en donde la playa se transforma en uno de los nuevos símbolos para la restauración de su imagen internacional.

\footnotetext{
${ }^{1}$ Según la Convención del Patrimonio Mundial, Cultural y Natural realizado en el marco de la $17^{\text {a }}$ Conferencia General de la UNESCO (1972), Patrimonio Cultural es definido como el conjunto de bienes muebles e inmuebles que pueden ser reconocidos de acuerdo con la importancia histórica, estética, etnológica y antropológica adquieren un valor único y de durabilidad representativa simbólica/material de una región.

${ }^{2}$ Cf. Mauricio de Abreu, Evolução Urbana do Rio de Janeiro.
} 
Como colonia portuguesa en los primeros años, la ciudad dependía del puerto y de las fortificaciones que protegían la bahía. En el siglo XIX, los baños de agua de $\operatorname{mar}^{3}$ comenzaron a utilizarse en Brasil como una técnica terapéutica contra enfermedades, lo permitió transformar la ciudad en un potencial centro de salud y baño. Al mismo tiempo, por las condiciones urbanas insalubres provocaron una serie de reformas urbanas - de saneamiento y embellecimiento - que transformaron por completo su fisonomía. Una de las más relevantes fue la remodelación urbana promovida por el alcalde Pereira Pasos entre los años de 1902 a 1906, con el apoyo y financiamiento del presidente Rodrigues Alves. El extenso programa incluyó la transformación e incluyeron la transformación de Río en una elegante ciudad de la "belle époque", considerando para ello; valores arquitectónicos franceses ${ }^{4}$, la apertura de nuevos trazados en el centro histórico y se rellenos en el borde costero que utilizaron la tierra extraída de los cerros, modificando sustancialmente su perfil litoral.

Según Iwata $^{5}$, con la modernidad, la ubicación residencial en la ciudad se convirtió en el símbolo de estatus social y estilo de vida, y los mejores distritos de la ciudad fueron sucesivamente impulsados en dirección a las playas del sur. De esta forma, Flamengo, Botafogo, URCA y algunos años más tarde, Leme, Copacabana, Ipanema y Leblon, fueron apropiados por las élites, cuyas hermosas casas se ubicaron primero a lo largo del paseo marítimo, y posteriormente a las principales arterias y las líneas del tranvía, que a lo largo del siglo XIX y siglo XX abrieron camino a la urbanización y la consecuente transformación del paisaje natural de la costa.

La ciudad de Viña del Mar, por su parte es una ciudad - hasta la actualidad - de menor escala que Río de Janeiro, tanto en su dimensión como en población, aun cuando es el balneario más relevante de la costa central chilena, configurándose como tal desde su proximidad a la capital de Santiago, pero por, sobre todo, por su condición de vecindad al puerto principal del país, Valparaíso.

Podemos afirmar que la distancia entre el centro de Río y Copacabana es similar a la distancia entre el puerto de Valparaíso y Viña del $\mathrm{Mar}^{6}$. En este sentido se funda, en parte por las mismas aspiraciones de la elite balnearia de finales del siglo XIX y principios del XX, de expandir su ambición inmobiliaria hacia sectores menos urbanizados y más exclusivos. De hecho, la ciudad se funda como una urbanización privada promovida en 1874 por los dueños del fundo que ahí existía, a través de la figura de Francisco Vergara, que propuso situar el trazado al interior entre una serie de elementos geográficos como: la cordillera de la costa, el cerro Castillo y el estero Marga-Marga ${ }^{7}$. Con ello la

\footnotetext{
${ }^{3}$ Patricia Farias, “A Praia Carioca”, 127.

${ }^{4}$ Eloisa Petti Pinheiro, Europa, França e Bahia, 148

${ }^{5}$ Nara Iwara y Vicente del Rio, "The image of the waterfront of Rio de Janeiro", 175

${ }^{6}$ Entre el centro de Río y Copacabana hay $10 \mathrm{~km}$, mientras entre Valparaíso y Viña del Mar hay 8km. En términos de población hay una diferencia radical teniendo Río cerca de 6 millones de habitantes, mientras Valparaíso (incluyendo Viña) no llega a los 600 mil.

${ }^{7}$ S/A, Decreto Oficial de la Fundación de Viña del Mar, 85.
} 
ciudad se proyectó como un suburbio residencial de Valparaíso ${ }^{8}$ respondiendo a aspiraciones sociales de elite. Estas condiciones fundacionales, se contradecían con el uso industrial de las playas más extensas, como Caleta Abarca y Acapulco, donde en la época se ubicaban muelles, industrias y maestranzas de diversa índole como extensión de las actividades comerciales del puerto ${ }^{9}$. Aun así, las actividades balnearias de la naciente elite se concentraron en la playa Miramar, que, a través de diversas instalaciones, se promovía el uso terapéutico de la playa ${ }^{10}$.

Se puede afirmar que existió una la tardía aproximación al borde costero por parte de la ciudad, lo que significó un borde bastante más fragmentado y poco homogéneo, si lo comparamos con el caso de Río, lo que se puede entender en parte por la geografía que los diferencia.

En el caso de Viña del Mar, también existieron una serie de leyes y obras que promovieron su condición balnearia, que incluyeron también la creación de infraestructura, urbanización y la construcción de un ambicioso plan de obras que trasformaron la ciudad mediterránea original y pintoresca, en una aspirante ciudad moderna.

Podemos decir que, si bien ambos casos son distintos en su configuración general, las condiciones de transformación desde el nacimiento mediterráneo al uso del litoral se debieron en gran parte a las aspiraciones sociales de la elite y a la masificación del uso de la playa como espacio social. Con ello se produjeron cambios importantes a nivel de intervenciones urbanas y arquitectónicas, que sintetizaremos en los próximos apartados.

\section{ESPECULACIÓN INMOBILIARIA Y RELLENOS URBANOS; LA CONSTRUCCIÓN DE LA FACHADA MARÍTIMA Y LA COSTANERA EN COPACABANA Y VIÑA DEL MAR}

Hasta fines del siglo XIX, Copacabana era un gran suburbio rural, zona de chacras y de residencias nobles, aislado de la ciudad. Como ha mencionado Elizabeth Dezouzarth Cardoso, en su libro clásico, "Histórias dos bairros: Copacabana", 11 , hasta 1870, tres caminos llevaban a Copacabana. Uno de ellos, y el más utilizado, empezaba en la calle Real Grandeza en Botafogo y pasaba sobre el Morro da Saudade, y bajaba por la ladera y calle del Barrozo, actual Tabajaras y Siqueira Campos. Sin embargo, es a partir de 1982, con la inauguración del Túnel de la Real Grandeza o Túnel Velho y la extensión de la línea de trenes de la Companía Jardim Botânico hacia Copacabana, es que comienza el proceso de urbanización, con la apertura de una serie de calles y el loteo del barrio por la Empresa de Construções Civis. Entre los años 1902 y 1906 fueron realizadas por el administrador de la ciudad,

\footnotetext{
${ }^{8}$ Cáceres, Booth y Sabatini. "Suburbanización y suburbio en el Chile: una mirada al Gran Valparaíso decimonónico 1820 $-1870 ", 151$.

${ }^{9}$ Urbina, M. Ximena. “Chalets y Chimeneas: Los primeros establecimientos industriales Viñamarinos, 1870 - $1920 ”, 173$.

${ }^{10}$ Von Schroeders, Teodoro. "Mis Memorias", 71.

${ }^{11}$ Elizabeth Dezouzarth Cardoso, História dos bairros: Copacabana, 24.
} 
Francisco Pereira Pasos, numerosas gestiones urbanas para el mejoramiento vial e higienización del centro de la ciudad. Para la zona sur de Río de Janeiro, Pereira Pasos promovió la vinculación de la Bahía de Botafogo con la playa de Copacabana a través de la apertura del Túnel Novo y de la apertura de la Av. Atlántica y la construcción de la costanera a lo largo de la playa en $1906^{12}$. Estas acciones serán las que determinarán el rol turístico de esta playa a inicios del siglo XX y permitirán su consolidación urbana hacia 1930.

En el caso de Viña del Mar, una de las transformaciones más relevantes es la llegada del tren desde Valparaíso en 1874 y que posteriormente las conectó con la misma capital en 1926. La llegada del tren creó diversas estaciones en las playas más concurridas, como Miramar y Recreo, lo cual además potenció la creación del camino plano entre ambas ciudades en 1901 y posteriormente el Puente Capuchinos de $1952^{13}$. De esta forma, la conectividad y la infraestructura vial y ferroviaria fueron detonantes de la incipiente urbanización de las zonas litorales entre Viña y Valparaíso ${ }^{14}$.

Entre los años treinta y cincuenta es cuando Copacabana sufre las transformaciones más radicales de su fachada urbana. Hasta los años 1920, el barrio estaba conformado, mayoritariamente, por residencias de uno y dos pisos (Figura 3). A lo largo de las siguientes décadas, fue varias veces reconstruida, dando paso a un proceso de densificación y verticalización. Según Cardeman y Cardeman $^{15}$, en los treinta surgieron edificios que variaban entre cuatro y diez, y que se concentraban principalmente en los alrededores del Copacabana Palace Hotel. Entre 1950 y 1960 los primeros edificios fueron sustituidos por otros más altos, después de la aprobación de la planta de zonificación para el barrio en 1946, cuyo límite de altura para los edificios pasaba a ser de 14 pisos $^{16}$. El barrio, en las siguientes décadas, siguió una lógica de construcción basada en esta planta de zonificación, hasta que en 1976 se aprueba una nueva ley que permite el aumento de altura de hasta 10,60 metros para la construcción de un zócalo para albergar estacionamientos ${ }^{17}$. En definitiva, se produce una verticalización de la fachada urbana de la playa, que se irá sustituyendo cada vez por edificios más altos, densificando el frente costero.

Según Vera Rezende ${ }^{18}$, esta continua transformación se debió, por un lado, por la ley del condominio en 1928 - Decreto n 5.481 de junio de 1928, que permitió la subdivisión de la propiedad

\footnotetext{
${ }^{12}$ Ciro Flamarión Cardoso y Paulo Henrique da Silva Araújo. Rio de Janeiro, 20.

${ }^{13}$ Silva. "Me llamo Viña del Mar", 81.

${ }^{14}$ Vicuña Mackenna. "La inauguración del camino de hierro entre Valparaíso y Viña del Mar”, 11.

${ }^{15}$ Cardeman y Cardeman, O Rio de Janeiro nas alturas, http://www.vitruvius.com.br/revistas/read/arquitextos/07.078/295 (consultado en 10 de enero 2018)

${ }^{16}$ Cardoso y Araújo, Rio de Janeiro, 201.

${ }^{17}$ Cardeman y Cardeman, O Rio de Janeiro nas alturas, http://www.vitruvius.com.br/revistas/read/arquitextos/07.078/295 (consultado en 10 de enero 2018)

${ }^{18}$ Vera Rezende, “Evolução da produção urbanística na cidade do Rio de Janeiro, 1900-1950-1965”, 43
} 
en edificios verticales con más de 5 pisos. Por otro lado, por el estimulo otorgado a la construcción por la crisis económica mundial y finalmente, por una mezcla de aspectos técnicos y disciplinares; como la introducción de hormigón armado y su difusión en la arquitectura brasilera de estos años. Pero sin duda que la mayor causa de esta constante construcción de la fachada urbana de Copacabana se debió a las presiones del turismo ${ }^{19}$ y el mercado inmobiliario que han situado a Copacabana como una de las playas más importantes reconocidas mundialmente.

El borde costero de Viña del Mar también fue un territorio de especulación, desde las iniciativas estatales a las privadas y desde las aspiraciones productivas a las balnearias. Todas ellas ejercieron una enorme presión urbana en el litoral, configurando un borde fragmentado ${ }^{20}$. Uno de ellos es el tramo conocido como la Av. Perú, donde la ciudad proyectó su crecimiento lineal a lo largo de la costa. La urbanización con sentido balneario del norte del estero Marga-Marga, en la Av. Perú, estuvo emprendida por la Sociedad Balneario de Viña del Mar, quien loteó los terrenos con fines residenciales $^{21}$. Donde además se ubicó estratégicamente el Casino de Viña de los arquitectos A. Risopatrón y R. Acuña (inaugurado el 31 de diciembre de 1930) 22 , que representó una obra creada para complementar las actividades del verano, generar una fuerte entrada de dinero para la municipalidad y configurar un foco de atención turístico y social. Al igual que en Río, pintorescas casas de dos o tres pisos se ubicaron en dicho borde y proyectaron la condición residencial de la zona, pero que su verticalización fue posterior y menos rápida, aun cuando la ley de venta por piso se dio en Chile solo diez años después que, en Brasil, en 1937.

La misma Sociedad que loteó los terrenos, realizó las primeras intervenciones sobre el borde costero al norte del estero Marga-Marga, a través de un malecón ${ }^{23}$ que permitió avanzar con terrenos sobre los arenales existentes, dejando un trozo de playa frente a ellos. Así lo corrobora una imagen de la época (Figura 4), donde se aprecia una costanera que, por el lado de la calle, se encontraba con luminaria y árboles en macetas, y por el lado de la playa, se encontraba un antepecho que dejaba algunas escaleras de bajada a la playa. Esta intervención proponía contener las fuertes marejadas que inundaban las casas del lugar.

\footnotetext{
${ }^{19}$ Habría que mencionar que en distintos momentos el Estado ha incentivado la construcción de hoteles en el país, como parte de un proyecto nacional de desarrollo. A partir de una serie de decretos de ley, ej. Decreto de ley 6761 (31 de julio de 1944); Decreto de ley 8055 (3 de mayo de 1945) etc., que objetivaban dar incentivos fiscales a las nuevas construcciones.

${ }^{20}$ Sobre la configuración fragmentada del borde costero de Viña del Mar revisar la tesis doctoral de una de las autoras: Cortés, "El balneario y la conquista formalizada del borde costero: Continuidades y fragmentos en Viña del Mar 1928 1963" (Tesis doctoral, Facultad de Arquitectura, Diseño y Estudios Urbanos, 2010)

${ }^{21}$ Ver "Plano de Conjunto - Playa y terrenos de la Sociedad - Playa Miramar". En S/A “Álbum de Viña del Mar”. Sociedad Balneario de Viña del Mar. Sociedad Imprenta y Litografía Universo, Valparaíso, 1913

22 "Junto con la llegada del año 1931 fue inaugurado en una inolvidable fiesta el Casino Municipal", 107.

${ }^{23}$ S/A. “Álbum e Viña del Mar".
} 
A pesar de la preocupación que existió en el momento por la desaparición paulatina de la playa, fue más importante contener las marejadas. Por ello, se construyó una defensa marítima para hacer frente a las constantes inundaciones producidas por las subidas del mar y del estero, anulando por completo las playas existentes.

Así lo corrobora el informe técnico ${ }^{24}$ de la creación del enrocado en la Av. Perú entre los años 1937 y $1944^{25}$. Esta obra consistió primero en la destrucción del malecón anterior, y se prosiguió con la construcción de un muro, para finalmente colocar el enrocado que enfrenta al mar.

La imagen de la obra muestra cómo definitivamente la playa quedó sepultada bajo el enrocado, y la Av. Perú despejaba el frente marino para las casas existentes (Figura 5). Esta obra resultó ser sólo una defensa marítima y no una habilitación de la Av. Perú como paseo público. No se proyectó ningún elemento de mobiliario urbano, barandas, arborización o luminaria. Una obra de infraestructura impresionante para la tecnología de la época, que destruyó casi un kilómetro de playa entre las calles 1 y 8 Norte.

En el caso de Río la acción fue totalmente contraria, y tendió a ampliar y valorizar la playa de Copacabana a través de la remodelación de la Av. Atlántica en los años 70. Esta había sido originalmente construida en 1906 durante las obras promovidas por el alcalde Pereira Pasos, momento que recibió el conocido juego de mosaicos negros y blancos, característicos de la tradición portuguesa, y posicionados en forma de ola. En 1919, la costanera fue ensanchada y reconstruida por el alcalde Paulo Frontin ${ }^{26}$. La reforma mencionada fue realizada entre los años 1968 a 1971, y consistió en ampliar la vía hacia un bulevar de doble calzada, de 80 metros de anchura. El objetivo fue facilitar el intenso movimiento vehicular generado por la creciente densidad demográfica enfrentada por la playa a partir de los años 40 y el gran flujo de turistas que a cada año buscaban este lugar. Tal construcción, realizada 10 años después de la fundación de Brasilia y de que Río dejara de ser la capital del país, fue posibilitada a través de una compleja operación de terraplén hidráulico que, a partir del espacio ganado al mar, triplicó el área de arena dedicada a los bañistas de la playa de Copacabana; haciendo posible, además, la construcción de una importante solución de aguas residuales ${ }^{27}$ (Figura 6). El proyecto de la Avenida quedó a cargo del arquitecto y paisajista Burle Max que intervino en la icónica imagen de la costanera, elaborando un nuevo diseño del paisaje de la calle. La intervención utilizó el empedrado de

\footnotetext{
${ }^{24}$ S/A. "Informe técnico de la Dirección de Obras Portuarias de Valparaíso", Viña del Mar, 1944. En Archivo Histórico Patrimonial de la I. Municipalidad de Viña del Mar.

${ }^{25}$ Este informe realizado por Luís Núñez, Inspector Fiscal de la Dirección de Obras Portuarias de Valparaíso, presentaba 42 imágenes con leyendas que describían la construcción del enrocado. La totalidad de ellas revela distintas instancias de la obra, exceptuando una que muestra una inundación. Probablemente esta última imagen justificaba la obra pública de defensa.

${ }^{26}$ Cardoso y Araújo, Rio de Janeiro, 211.

${ }^{27}$ Nara Iwara y Vicente del Rio, “The image of the waterfront of Rio de Janeiro", 177.
} 
mosaico portugués, manteniendo el dibujo en forma de olas del mar en la vereda más próxima a la playa y creando un diseño distinto en las otras veredas. La nueva composición del paisaje de la playa de Copacabana y junto con la densa fachada marítima, producto de la voracidad inmobiliaria de los años 40 y 50, se transformó en la nueva imagen de la cultura de la playa (Figura 7).

Por otro lado, y a pesar de todo, la Av. Perú en Viña del Mar se configuró y ha permanecido hasta hoy en día como un espacio, que, si bien carece de proyecto urbano y arquitectónico, que ha logrado configurarse como el paseo más importante de la ciudad. Cada año dicho enrocado resiste las marejadas que aún persisten en devolver la playa a su estado original y enfatizan la condición natural de los terrenos ganados al mar. La verticalización de la fachada marítima se dio frente al enrocado, solo en una oportunidad (y no reiteradas veces como en Río) y dependió principalmente de la explosión inmobiliaria a partir de Decreto de Fuerza de Ley No 2, "Plan Habitacional de Viviendas Económicas de 1959"28, que, controlando el metraje cuadrado de la vivienda, garantizaba la rebaja de impuestos. El DFL2 tenía un fin social, pero fue aprovechado por la gestión inmobiliaria, para dar una segunda vivienda a quienes la necesitarían estacionalmente. Los edificios nacidos al amparo de dicha ley, consolidaron la imagen moderna de la ciudad turística en varios puntos, incluyendo la Av. Perú. Dicha etapa presentó una contradicción entre la promoción del balneario a través de la especulación inmobiliaria del borde costero y la protección de éste mismo, a través de otros instrumentos legislativos, como la Ley Lorca de 1962, que obtuvo un empréstito para realizar obras de progreso y estuvo destinada realizar las expropiaciones para la habilitación turística de la playa que continuaba había el norte del enrocado antes descrito ${ }^{29}$. Dicha ley tuvo asociado un proyecto urbano llamado "Conjunto Muelle Población Vergara"30, (construido parcialmente) el cual consistía en nueve torres de 15 pisos de altura frente al mar y colocadas sobre un parque abierto. Esta altura que no se replicó en la Av. Perú, que solo alcanzó los 10 pisos de altura promedio, con edificación aislada (Figura 8).

\footnotetext{
${ }^{28}$ Decreto de Fuerza de Ley de 31-7-1959. Sobre Plan Habitacional de Viviendas Económicas. Extracto del Título I "Se considerarán viviendas económicas, para los efectos del presente decreto con fuerza de ley, las que se construyan en conformidad a sus disposiciones, tengan una superficie edificada no superior a 140 metros cuadrados por unidad de vivienda". Extracto del Título II, "Los recibos de dinero correspondientes a precio en contratos de construcción y compraventa de viviendas económicas estarán exentos del impuesto". En http://www.sii.cl/pagina/jurisprudencia/proley/Decretro2. htm, (consultado el 7-09)

${ }^{29}$ Lorca, Gustavo. “Cuatro años de Administración Municipal Comunal (Viña del Mar), 1958-1962”, 20.

${ }^{30}$ Según Plano “Conjunto Muelle población Vergara”, Bolton, Larraín, Prieto y Lorca; Oficina C. Cruz, Munizaga, R. Cruz; O cina Echenique y José Cruz; Oficina Goldehiberg y Mitrovic; Oficina J. Larraín V. , O. Larraín E., J. Larraín E. Plano No1, 25 de enero de 1960 y modificación de 4 de mayo de 1960.
} 
4 PATRIMONIO EDILICIO Y NATURAL EN EL BORDE COSTERO, UNA TAREA PENDIENTE

Podemos concluir que, aun cuando siendo casos muy diversos en condiciones climáticas y geográficas, como en la configuración urbana, la historia de ambas ciudades puede ser entendida bajo algunas premisas del desarrollo balneario costero en el siglo XX. Por un lado, la configuración inicial mediterránea, será modificada por un acceso paulatino a la playa focalizado en las terapias de salud y la prescripción médica de las estancias marinas. Por otro lado, configuración preferentemente lineal a lo largo del litoral ha sido la forma de desarrollo urbano, que ha ido conquistando nuevos territorios y playas, identificado dichos espacios como el potencial fundamental en términos de espacio público, como de especulación inmobiliaria. Finalmente, aun cuando las diferencias son evidentes en términos de calidad dicho espacio, tanto en Viña del Mar como en Copacabana existieron modificaciones importantes e incluso violentas con el espacio natural, a través de obras de infraestructura de conectividad (como túneles, líneas férreas, caminos etc.) pero por sobre todo con obras de drenaje, aterramiento, enrocados etc, que modificaron de manera sustancial la fisonomía natural del borde costero.

En dicho contexto, la playa exige un conjunto continuo e integrado de políticas y programas de diseño urbano. Las playas de Río de Janeiro y de Viña del Mar deben dejar de ser vistas solo como un tema de "construcción natural" de folletos turísticos y postales, para ser entendidas como áreas estratégicas para el desarrollo de la ciudad, donde existe una urgencia en la valorización patrimonial de sus fachadas, costaneras u otros elementos (como el caso del Muelle Vergara), como de sus condiciones naturales, que deben ser preservados como acervo cultural y social de estos balnearios. 


\section{REFERENCIAS}

Abreu, Mauricio. 2013. Evolução Urbana do Rio de Janeiro. Rio de Janeiro: Instituto Pereira Pasos.

Andreatta, Verena; Chavari, Maria Pace; Rego, Helena. O Rio de Janeiro e sua orla: história, projetos e identidade carioca. Coleção Estudos Cariocas, n. 20091201. Rio de Janeiro: Instituto Pereira Passos, 2009

Cardeman, David; Goldfeld Cardeman, Rogério. O Rio de Janeiro nas alturas: A verticalização da cidade. Arquitextos, São Paulo, ano 07, n. 078.02, Vitruvius, nov. 2006. http://www.vitruvius.com.br/revistas/read/arquitextos/07.078/295 (consultado en 10 de enero 2018)

Cardoso, Ciro Flamarión y da Silva Araújo, Paulo Henrique. 1992. Rio de Janeiro. Madrid: Editorial MAPFRE.

Cáceres, Booth y Sabatini. 2002. "Suburbanización y suburbio en el Chile: una mirada al Gran Valparaíso decimonónico (1820 - 1870)". En Revista Archivum, Revista del Archivo Histórico Patrimonial de la I. Municipalidad de Viña del Mar, Año III, Nº4, (2002): 151-164.

Decreto Oficial de la Fundación de Viña del Mar, 1874. En Silva, Roberto. "Me llamo Viña del Mar". Talleres gráficos EDEVAL, Viña del Mar, 1974.

Farias, Patricia. A praia carioca, da colônia aos anos 90: uma(s) história(s). Contracampo. Niterói, n4, pp.125-144, 2000.

Iwata, Nara; Del Rio, Vicente. The image of the waterfront in Rio de Janeiro. Urbanism and Social Representation. Journal of Planning Education and Research, 2004, n24, pp.171-183.

Perez, Julia Rey. O passeio de Copacabana como patrimônio e paisagem cultural. Revista UFG. Goiânia: Pró-Reitoria de Extensão e Cultural, Julio 2010, n8, pp.106-116

Lorca, Gustavo. "Cuatro años de Administración Municipal Comunal (Viña del Mar), 1958-1962”. Ilustre Municipalidad de Viña del Mar, Viña del Mar, 1962. pp. 20.

Machado, Marcelo de Barros Tomé. 2008. A Modernidade no Rio de Janeiro: construção de um cenário para o turismo. Rio de Janeiro: Prefeitura Municipal do Rio de Janeiro.

Maderuelo, Javier. 2005. El Paisaje: Génesis de un Concepto. Madrid: Abada Editores.

Rezende, Vera. 1999. "Evolução da produção urbanística na cidade do Rio de Janeiro, 1900-19501965". En: Urbanismo no Brasil, 1885-1965. Coordinado por Maria Cristina da Silva Leme, pp. 3970. São Paulo: Studio Nobel/FAUUSP/FUPAM.

Pinheiro, Eloisa Petti. 2011. Europa, França e Bahia. Difusão e adaptação de modelos urbanos. (Paris, Rio, Salvador). Salvador: EDUFBA

S/A. “Álbum e Viña del Mar”. Sociedad Balneario de Viña del Mar. Sociedad Imprenta y Litografía Universo, Valparaíso, 1913.

S/A, "Junto con la llegada del año 1931 fue inaugurado en una inolvidable fiesta el Casino Municipal". En Basulto L. Renato. "75 años de Viña del Mar: 1879-1954”. Imprenta y Litografía Sánchez, Valparaíso, 1954. 
Silva, Roberto. “Me llamo Viña del Mar”. Talleres gráficos EDEVAL, Viña del Mar, 1974.

Unesco. Convenção para a proteção do Património Mundial, Cultural e Natural. Paris: Unesco, 1972. Disponível em: https://whc.unesco.org/archive/convention-pt.pdf Consultado: 10 de julho de 2018.

Urbina, M. Ximena. "Chalets y Chimeneas: Los primeros establecimientos industriales Viñamarinos, 1870 - 1920”. En Revista Archivum, Revista del Archivo Histórico Patrimonial de la I. Municipalidad de Viña del Mar, Año IV, N5, (2003): 173-196

Vicuña Mackenna, Benjamín. "La inauguración del camino de hierro entre Valparaíso y Viña del Mar". La Unión 5 de noviembre de 1922. En Vicuña Mackenna, Benjamín. "Crónicas Viña Marinas". Talleres Gráficos Salesianos, Valparaíso, 1931.

Von Schroeders, Teodoro. "Mis Memorias”. Viña del Mar, 1915. 


\section{LISTA DE FIGURAS}

Figura 01 - Avenida Perú, al fondo Muelle Vergara, 2006. Fuente: Flickr.com/Francisco, editado por los autores a escala de gris. Disponible en: https://www.flickr.com/photos/pino_aromatico/335454967. Consultado en 1 de noviembre de 2018.

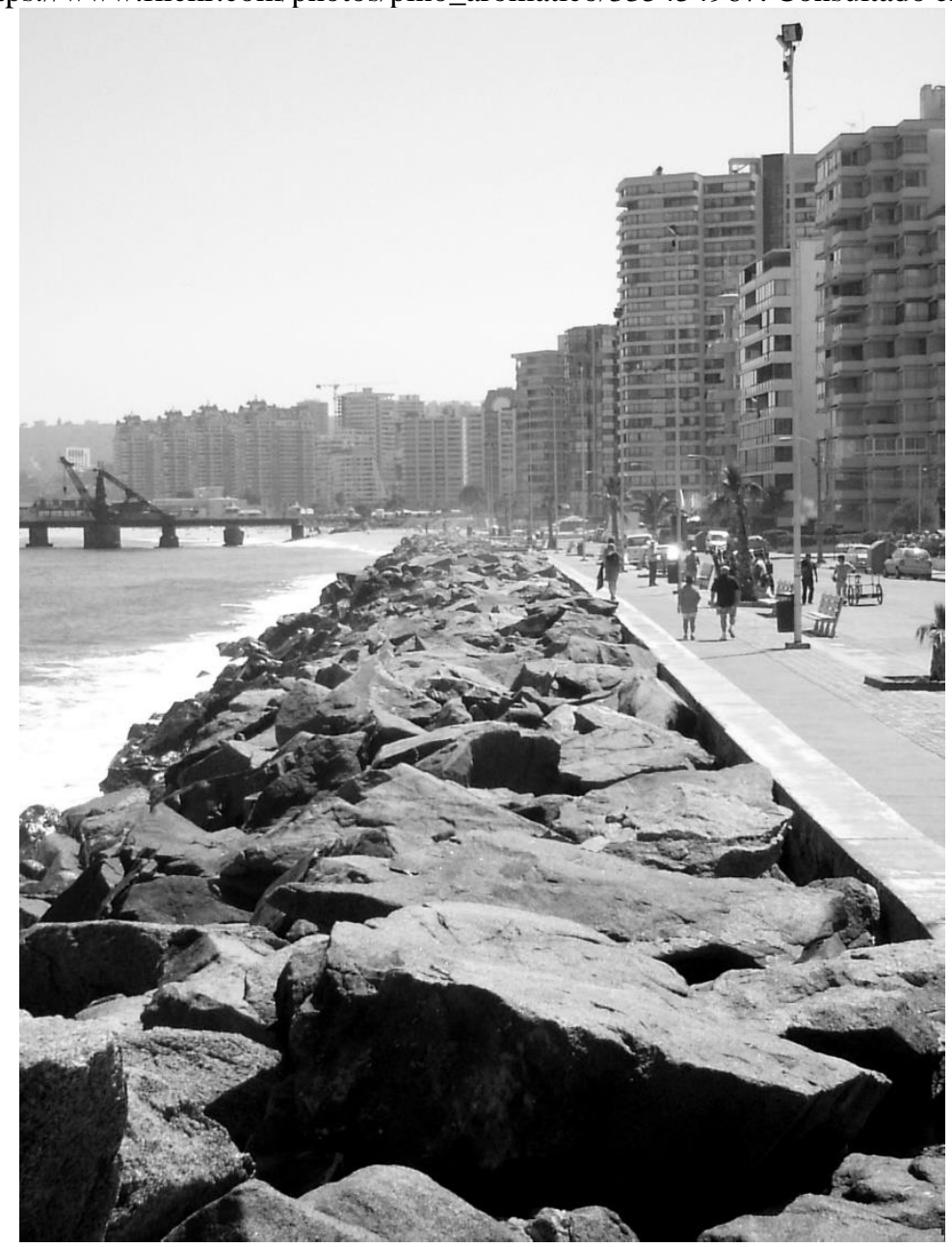

Figura 02 - Vista Panorámica Rio de Janeiro, 2004. Fuente: FreeImages.com/SpelaAndolsek, editado por los autores a escala de gris. Disponible en: https://es.freeimages.com/photo/south-america-1-1491804 Consultado en: 1 de noviembre de 2018.

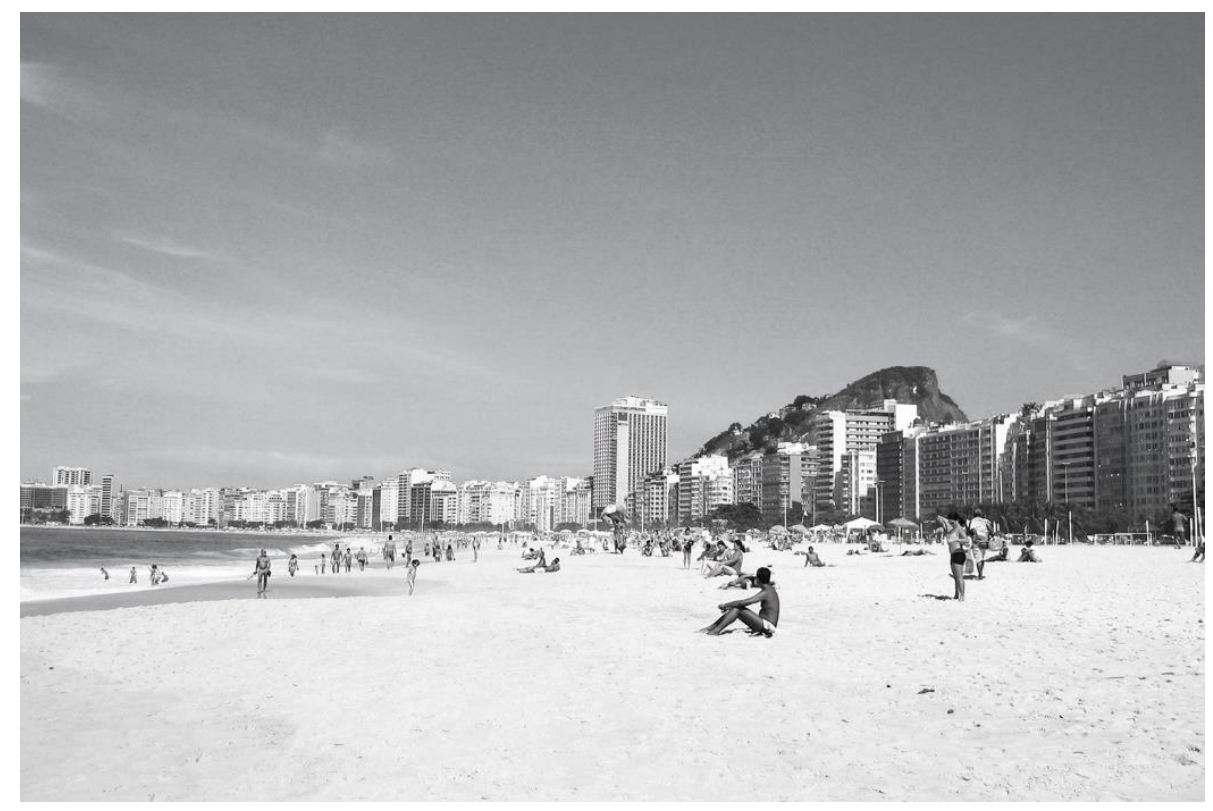




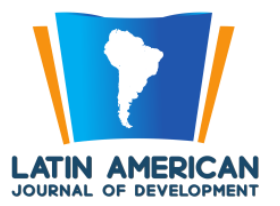

Figura 03 - Avenida Atlántica, Copacabana - Leme. al fondo la Piedra de Gávea, 1929. Fuente: Archivo General de la ciudad de Rio de Janeiro.

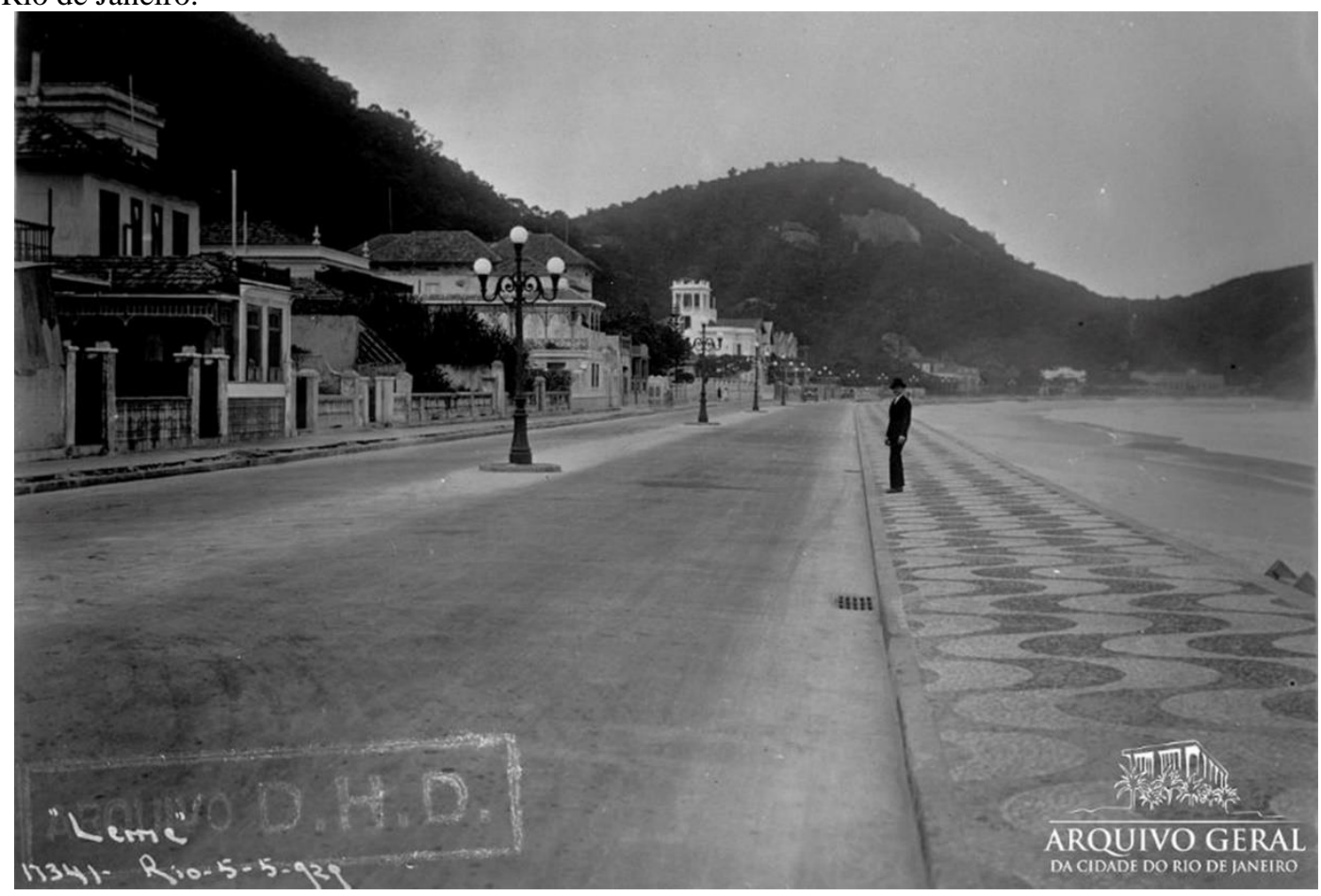

Figura 04 - Vista de la antigua playa de la Av. Perú, antes de la construcción del enrocado, hacia 1930. Fuente: Archivo Patrimonial de Viña del Mar

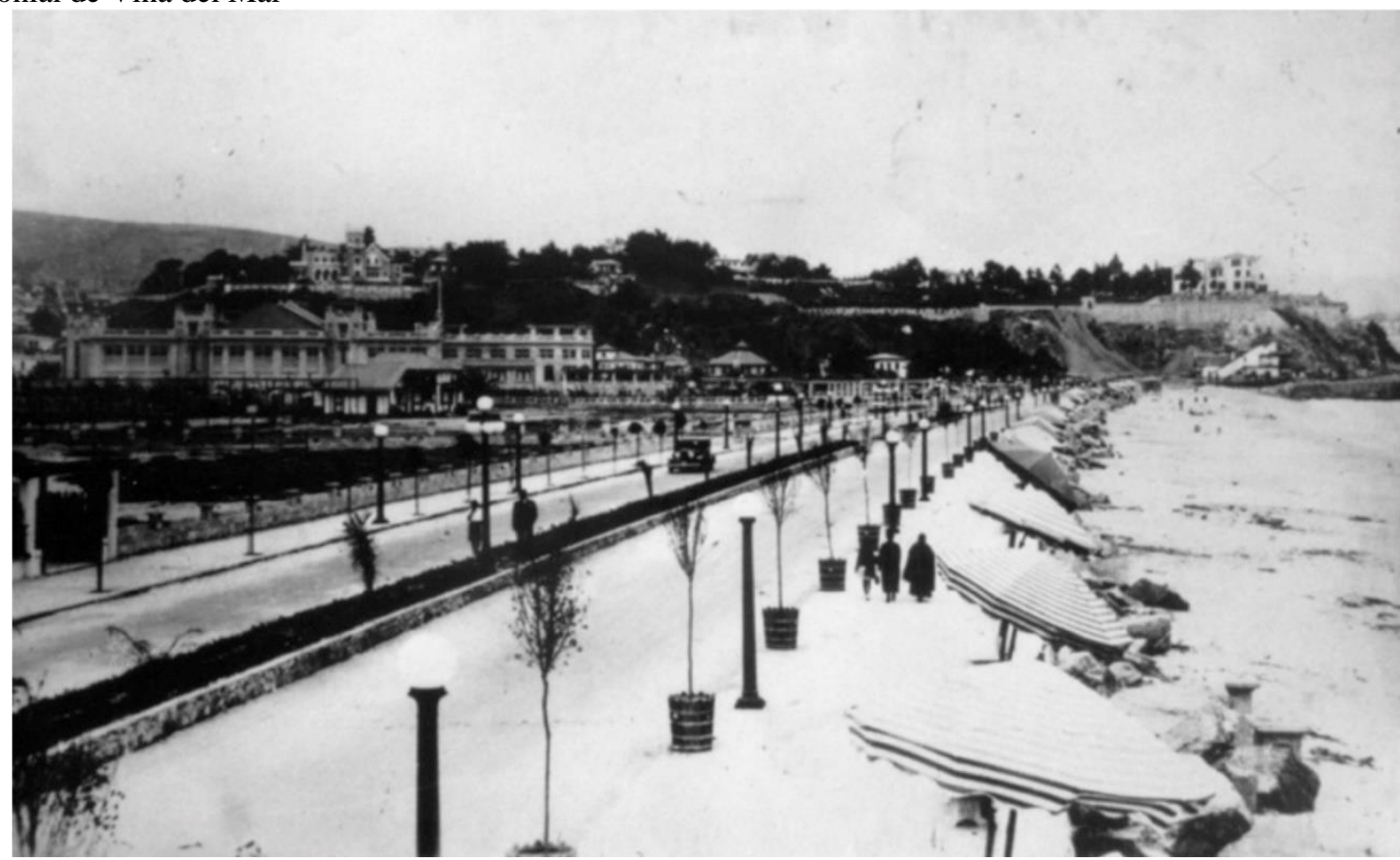


Figura 05 - "Vista parcial del enrocado de Av. Del Mar, colocado en 1915 por la firma Pearson and Son y completado con 40.188,582 ton. Colocadas entre 1937 a 1939 por el ex-departamento de Obras Marítimos entre 1 y 8 Norte. Fuente: "Informe Técnico de la Dirección de Obras Portuarias de Valparaíso", 1944. Archivo Histórico Patrimonial de Viña del Mar.

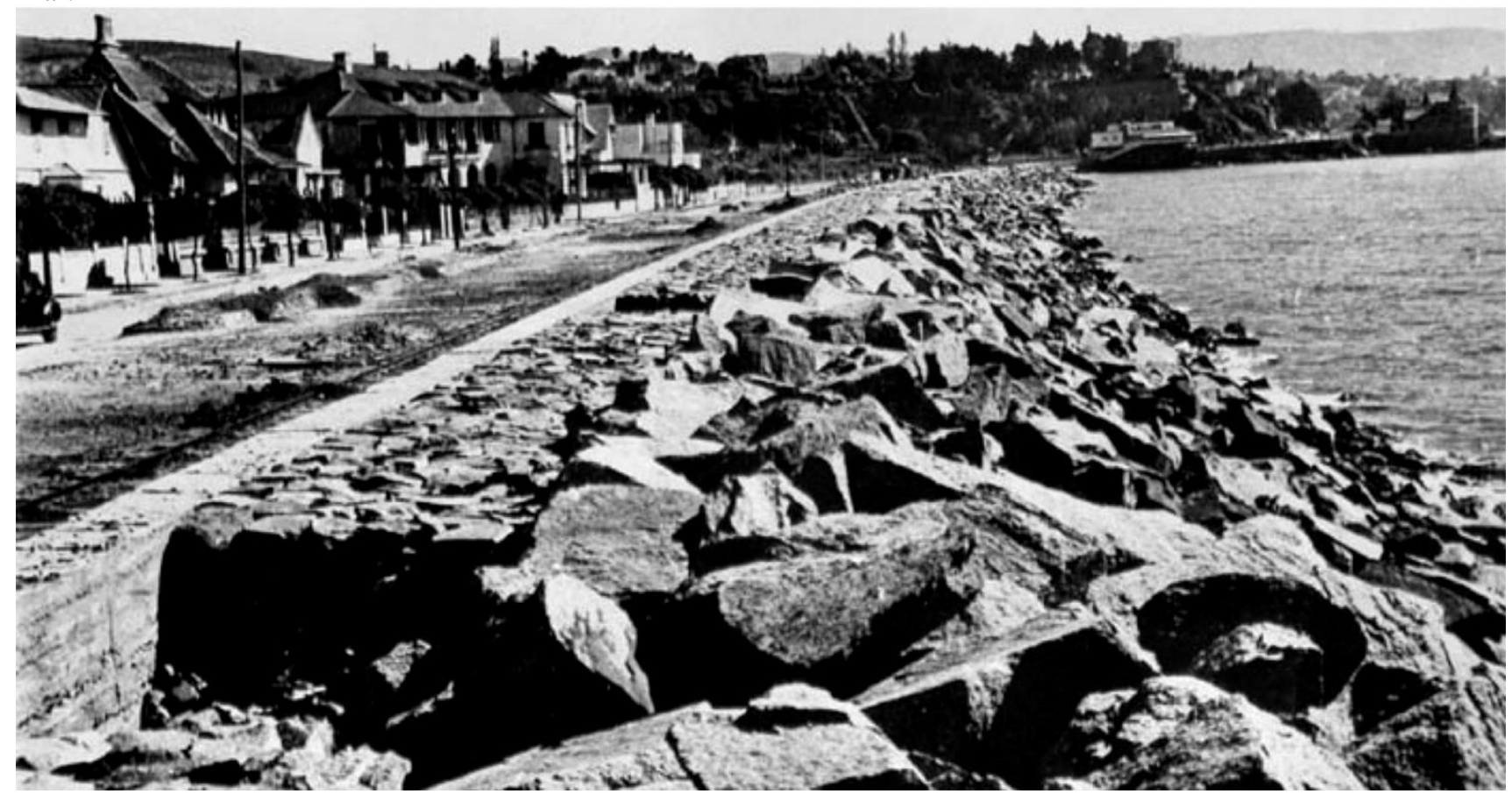

Figura 06 - Obras de ensacamento de la Avenida Atlántica, Copacabana. Al fondo, cerro Cantagalo, 1970. Fuente: Archivo General de la ciudad de Rio de Janeiro.

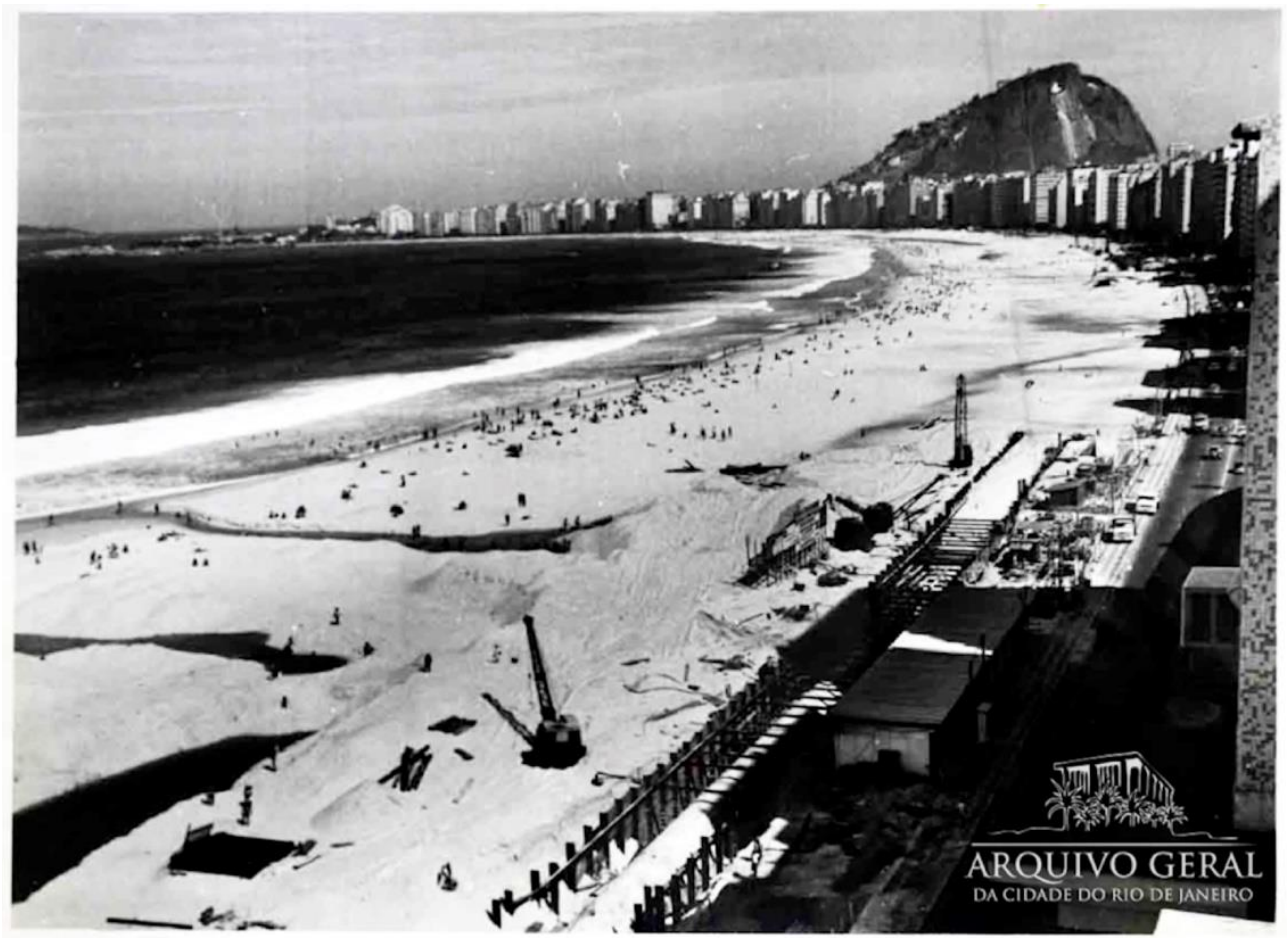




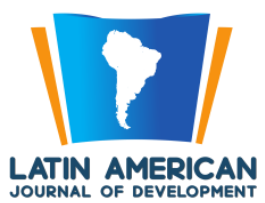

Figura 07 - Vista Aérea Avenida Atlántica, 1970. Fuente: Archivo General de la ciudad de Rio de Janeiro. Fotógrafo: Manuel de Souza Rocha.

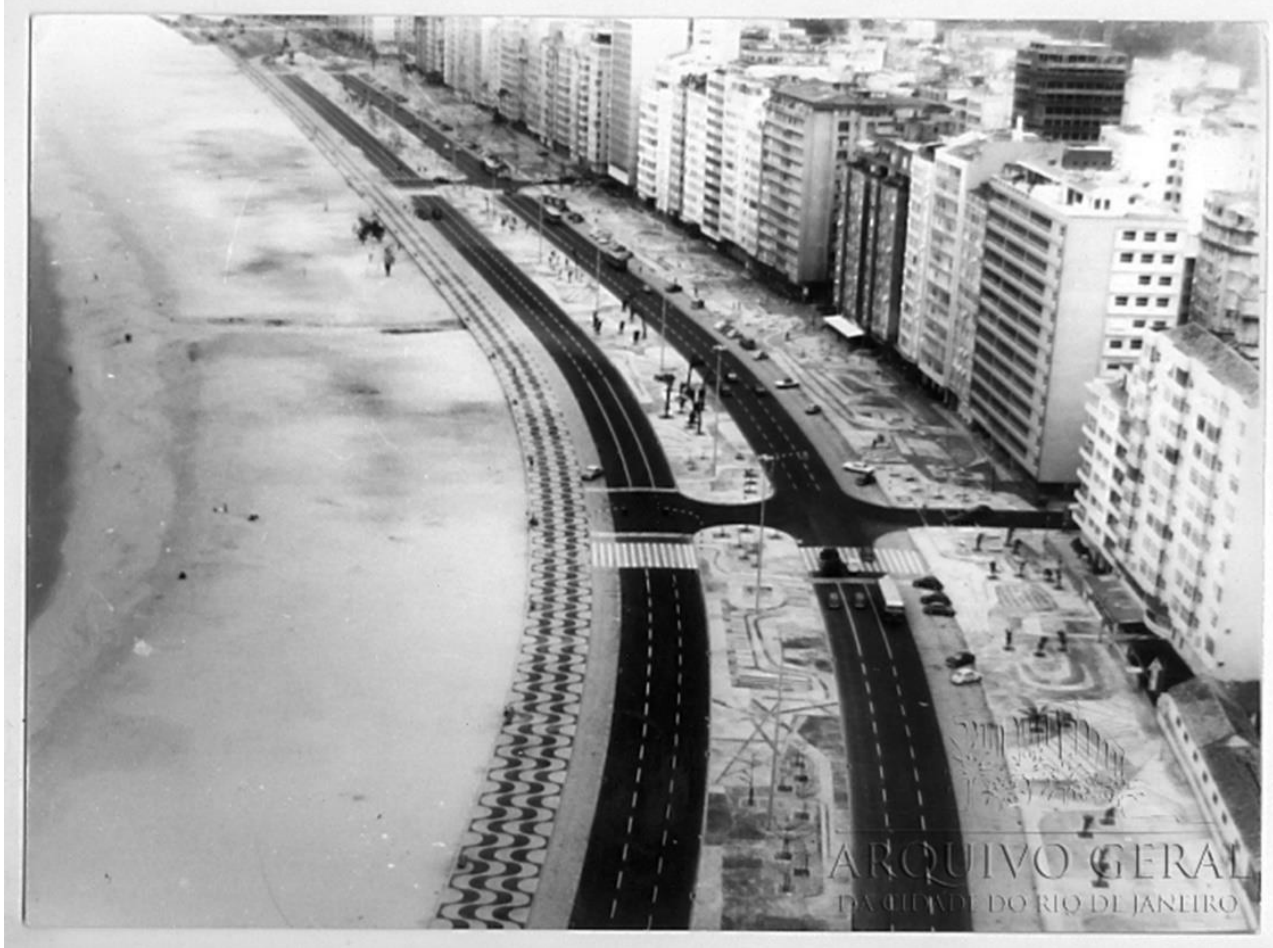

Figura 08 - Vista aérea de Viña del Mar hacia Avda. Perú. En primer plano Edificio Acapulco en construcción y tras él, la desaparecida piscina de 8 Norte. Fuente: En Archivo Histórico Patrimonial de la Ciudad de Viña del Mar. Sin fecha.

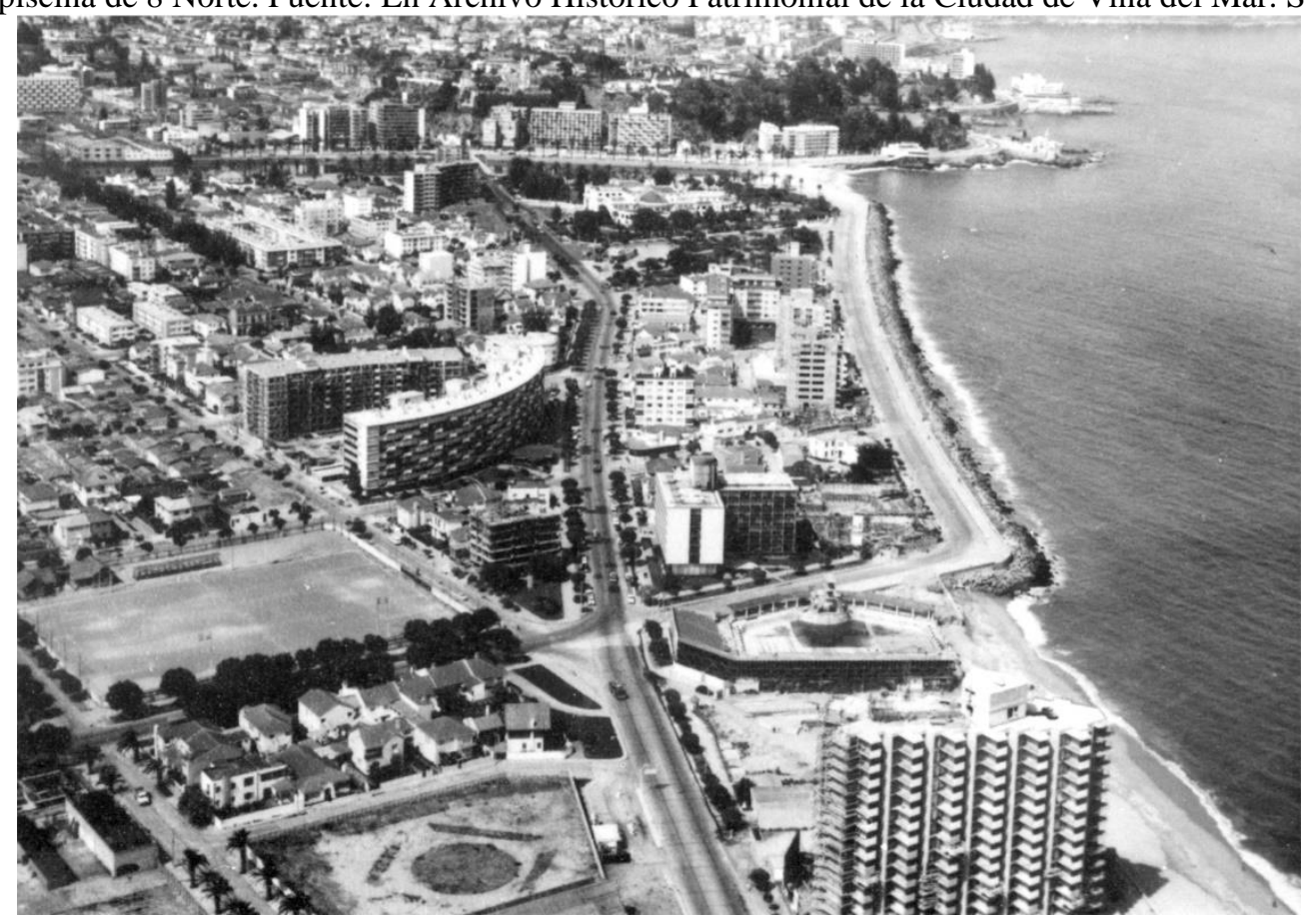

